Bryn Mawr College

Scholarship, Research, and Creative Work at Bryn Mawr

College

Physics Faculty Research and Scholarship

Physics

1984

\title{
Nuclear-Spin-Relaxation Study of Internal Motion in Two Organic Molecular Solids
}

Alfonso M. Albano

Bryn Mawr College, aalbano@brynmawr.edu

Peter A. Beckmann

Bryn Mawr College, pbeckman@brynmawr.edu

M. E. Carrington

E. E. Fisch

F.A. Fusco

See next page for additional authors

Let us know how access to this document benefits you.

Follow this and additional works at: http://repository.brynmawr.edu/physics_pubs

Part of the Physics Commons

\section{Custom Citation}

A.M. Albano et al. Phys. Rev. B 30, 2334 (1984).

This paper is posted at Scholarship, Research, and Creative Work at Bryn Mawr College. http://repository.brynmawr.edu/physics_pubs/37

For more information, please contact repository@brynmawr.edu. 
Authors

Alfonso M. Albano, Peter A. Beckmann, M. E. Carrington, E. E. Fisch, F. A. Fusco, A. E. O'Neill, and Mary E. Scott 


\title{
Nuclear-spin-relaxation study of internal motion in two organic molecular solids
}

\author{
A. M. Albano, P. A. Beckmann, M. E. Carrington, ${ }^{*}$ E. E. Fisch, F. A. Fusco, ${ }^{\dagger}$ A. E. O’Neill, ${ }^{\ddagger}$ and M. E. Scott \\ Department of Physics, Bryn Mawr College, Bryn Mawr, Pennsylvania 19010
}

(Received 17 November 1983; revised manuscript received 16 April 1984)

\begin{abstract}
We investigate proton spin-lattice relaxation in two powdered organic molecular solids with reorienting methyl $\left(\mathrm{CH}_{3}\right)$ and tert-butyl $\left[\mathrm{C}\left(\mathrm{CH}_{3}\right)_{3}\right]$ groups: 3,5-di-tert-butylphenol (DTBP) and 1,3,5-tri-tert-butylbenzene (TTBZ). The temperature and Larmor frequency dependence of the relaxation rate is considerably more complicated than would be expected on the basis of random reorientations of the $\mathrm{CH}_{3}$ and $\mathrm{C}\left(\mathrm{CH}_{3}\right)_{3}$ groups. The spectral densities describing the relaxation are broader, and show more structure, than those customarily used to interpret relaxation data. Assuming that the spectral densities may be expressed as linear superpositions of Lorentzians, we are able to obtain a single, nonexponential correlation function which leads to satisfactory fits of the data. Assuming also the validity of the Arrhenius relation, $\tau=\tau_{\infty} \exp (E / k T)$, with a constant $\tau_{\infty}$, we find that the barriers to rotation for the $\mathrm{CH}_{3}$ and $\mathrm{C}\left(\mathrm{CH}_{3}\right)_{3}$ groups may be characterized by a distribution of activation energies consisting of a wide background and one (DTBP) or two (TTBZ) $\delta$ functions.
\end{abstract}

\section{INTRODUCTION}

Molecular reorientation is characterized by a spectral density function $J(\omega, \tau)$, where $\omega$ is a frequency characteristic of the measuring apparatus and $\tau$ is a correlation time which characterizes the molecular motion. A detailed understanding of motional processes is a difficult problem because experiments rarely yield sufficient information to test realistic models of the dynamics. Nuclear magnetic resonance and, in particular, nuclear-spin relaxation is one of the best techniques to study these processes. It allows an investigation of motions in the vicinity of $\omega \sim \tau^{-1}$ for some diffusional processes, some anisotropic whole molecule reorientational processes, and most types of intramolecular reorientations. In this case, $\omega=\gamma B$ is the nuclear Larmor angular frequency where $\gamma$ is the magnetogyric ratio of the nucleus under investigation and $B$ is the applied magnetic induction. ${ }^{1}$ The frequency $\tau^{-1}$, which characterizes the molecular motion, can be varied by changing the temperature $T$. This is less direct than varying $\omega$ since it involves assumptions about the relationship between $\tau$ and $T$. Although the information obtained is incomplete, nuclear-spin relaxation seems the best way to study molecular dynamics in the frequency range corresponding to intramolecular motion.

Whole molecule diffusion and isotropic reorientation in isotropic liquids are two examples of dynamical processes which have been understood for some time and are well characterized by a Lorentzian spectral density, $J(\omega, \tau)=2 \tau /\left(1+\omega^{2} \tau^{2}\right)$. This follows from the assumption that the motion is random and isotropic. ${ }^{1}$ The case of anisotropic reorientation is more difficult, not so much from the theoretical point of $v^{2} w^{2}$ but from the point of linking the observed relaxation rates with the parameters in the theoretical models. If anisotropic reorientation is superimposed on diffusional processes, or intramolecular reorientations are superimposed on anisotropic whole molecule reorientation, then the problem becomes very difficult. In general, the greatest difficulty in using liquids to study these motions is that the region $\omega \sim \tau^{-1}$ is difficult to probe since the short correlation-time limit usually dominates $\left(\omega<<\tau^{-1}\right)$.

We have been investigating this molecular dynamics problem by working in solids whose structure is sufficiently simple to allow only certain limited well-defined motions to occur. To be more precise, we have concentrated on proton nuclear-spin relaxation in a series of organic molecules containing tert-butyl groups on a benzene ring. ${ }^{3-7}$ Two examples which are discussed in this paper are shown in Fig. 1. They are 3,5-di-tert-butylphenol-OD (DTBP) in which the $\mathrm{OH}$ proton has been replaced by a deuteron and 1,3,5-tri-tert-butylbenzene (TTBZ). These systems have the advantage that there are only two welldefined motions: the reorientations of the methyl $\left(\mathrm{CH}_{3}\right)$ groups and the tert-butyl $\left[\mathrm{C}\left(\mathrm{CH}_{3}\right)_{3}\right]$ groups about their threefold axes. The methyl reorientation is superimposed on the tert-butyl reorientation. Since spin-spin relaxation is orders of magnitude more efficient than spin-lattice relaxation (unlike the situation in a liquid), spin diffusion is very effective and all protons (i.e., both ring and tert-butyl protons) relax at the same rate. Also, all three regions $\tau<<\omega^{-1}, \sim \omega^{-1}$, and $\gg \omega^{-1}$ are accessible since temperature can be varied over a wide range. The geometric simplicity of the molecular systems, coupled with the ability to vary both $\omega$ and $\tau$ (via the temperature $T$ ), results in the possibility of learning a great deal about $J(\omega, \tau)$.

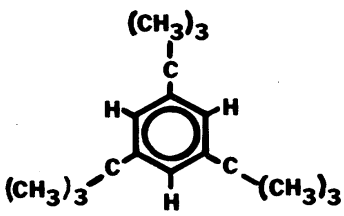

(a)

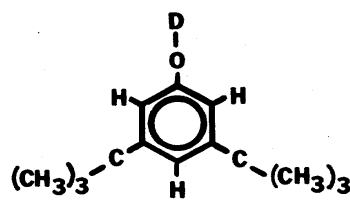

(b)
FIG. 1. Schematic diagrams of the molecules (a) 1,3,5-tritert-butylbenzene (TTBZ) and (b) 3,5-di-tert-butylphenol-OD (DTBP). 
This, in turn, allows one to test models for molecular motion and ultimately to test models for intramolecular and intermolecular electrostatic potentials.

In an investigation of the temperature dependence of the proton spin-lattice relaxation rate $R$ in solid DTBP at $\omega / 2 \pi=30 \mathrm{MHz}$, we found that none of the well-known phenomenological spectral density functions $J(\omega, \tau)$ which are used to describe relaxation processes would fit the data. ${ }^{6}$ Later, we repeated the measurements of 8.5 $\mathrm{MHz}$ and a new $J(\omega, \tau)$ based on quite general factors dictated by the experiment was developed. ${ }^{4}$ Although this new $J(\omega, \tau)$ satisfactorily fit the Larmor frequency and temperature dependence of the relaxation data simultaneously, it was difficult to relate the parameters which characterize it to parameters which characterize a distribution of correlation times (or of activation energies) for methyl and tert-butyl reorientation. We have extended the measurements in DTBP to $53 \mathrm{MHz}$ and they are shown along with the previous data ${ }^{4,5}$ in Fig. 2. The interesting surprise at $53 \mathrm{MHz}$ is the appearance of a steep slope in $\ln (R)$ versus $T^{-1}$ at the lowest temperatures. This did not occur at 8.5 or $30 \mathrm{MHz}$. This means that our recently developed spectral density ${ }^{4}$ is inadequate, at least in the simplest form used to fit the lower-frequency data. We have now adopted a completely new approach in the search for a spectral density function which would

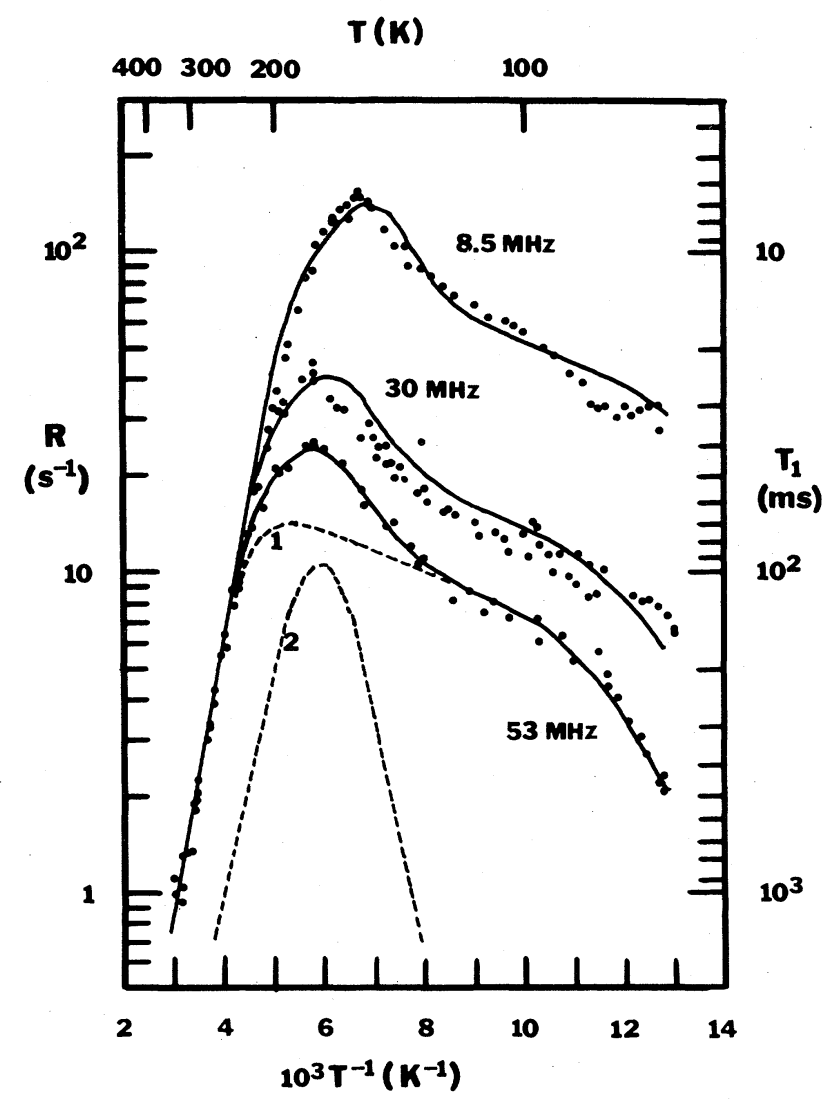

FIG. 2. Spin-lattice relaxation rate $R$ vs temperature $T$ in DTBP [shown in Fig. 1(b)]. The solid lines are fits to Eqs. (2), (5), and (6) with parameters given in the text. For the $53-\mathrm{MHz}$ solid line, the two terms in Eq. (5) are indicated by the two dashed lines.

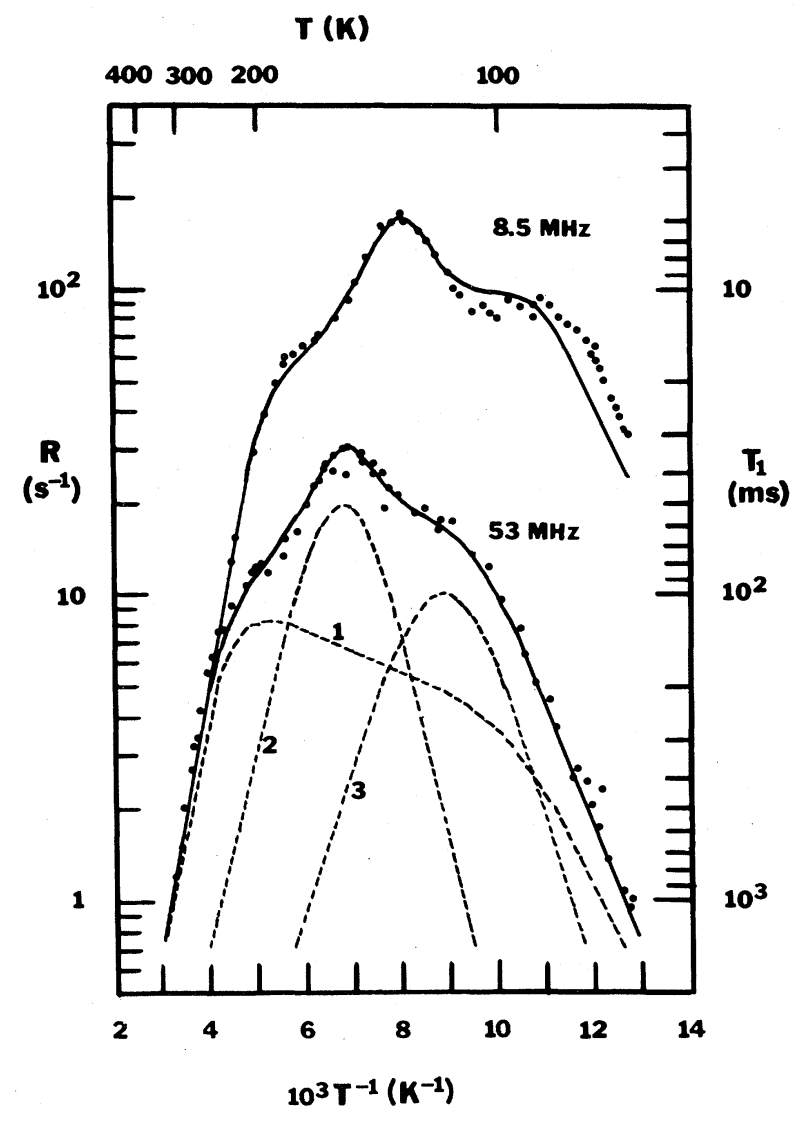

FIG. 3. Spin-lattice relaxation rate $R$ vs temperature $T$ in TTBZ [shown in Fig. 1(a)]. The solid lines are fits to Eqs. (2), (5), and (6) with parameters given in the text. For the $53-\mathrm{MHz}$ solid line, the three terms in Eq. (5) are indicated by the dashed lines.

predict this interesting low-temperature, high Larmor frequency behavior of the relaxation rate. We have found that we are able to start with simple distributions of activation energies which predict all the salient features of the observed rates. The same phenomenon which manifests itself in DTBP occurs in TTBZ at both 8.5 and 53 $\mathrm{MHz}$ as shown in Fig. 3. We use TTBZ as another example of our approach since we want to point out that some elements of our theory may be quite general. The presence of the OD group in DTBP but not TTBZ makes the two molecules potentially quite different in terms of the intermolecular local environment of the tert-butyl groups. We will discuss these matters in more detail after presenting some details of the relaxation measurements.

\section{EXPERIMENTAL}

Proton spin-lattice relaxation rates $R$ were measured in powdered 1,3,5-tri-tert-butylbenzene (TTBZ) and 3,5-ditert-butylphenol (DTBP) as a function of temperature $T$ at Larmor frequencies of $\omega / 2 \pi=8.5$ and $53 \mathrm{MHz}$. The data are shown in Figs. 2 and 3. In addition, we show previously reported data in DTBP at $8.5 \mathrm{MHz}$ (Ref.4) and $30 \mathrm{MHz}$ (Ref. 5) in Fig. 2. The lowest-temperature 
8.5- $\mathrm{MHz}$ results are new. The rates were measured with a $\pi-t-\pi / 2-t_{R}$ pulse sequence using a spin-lock CPS-2 spectrometer operating at either 8.5 or $53 \mathrm{MHz}$. The repetition period was $t_{R}>8 R^{-1}$. Temperature was controlled by a flow of reheated cold nitrogen gas and measured with a calibrated copper-constantan thermocouple. The free induction decay following the $\pi / 2$ pulse was gated and averaged by a EG\&G Parc Model 160 Boxcar Analyzer with a Model 165 Gated Integrator Module plug-in. This signal was further averaged by a Hewlett-Packard Model 5328A Universal Counter which simultaneously monitored the time $t$ between the $\pi$ and $\pi / 2$ pulses. The signal-versus- $t$ data was sent to a $32 \mathrm{~K}$-kbyte (1 kbyte $\equiv 1024 \times 8$ binary digits) Commodore PET microcomputer on a IEEE-488 interface bus for storage, handling, and computation of $R$. $R$ was determined from a plot of the natural logarithm of the difference signal versus $t$. The difference signal is the departure of the signal at time $t$ from the equilibrium signal. The statistical uncertainties in $R$ are small, usually less than $1 \%$, but there are systematic errors present, which are associated mainly with the determination of the equilibrium signal $\left(t \gg R^{-1}\right)$. The reproducibility error in the newly reported 8.5- and 53- $\mathrm{MHz}$ data can be judged from the scatter in Figs. 2 and 3 and is about 5\%. The previously reported measurements in DTBP at $30 \mathrm{MHz}$ (Ref. 5) predate the present automatic data acquisition system and are discussed below.

There are two procedures for preparing the samples for $R$-versus- $T$ measurements, both of which have been used extensively in this laboratory. One technique, not used in the present study, is the normal freeze-pump-thaw technique where the sample is sealed off either under a vacuum or with an inert gas. This has the advantage that, excluding photoreactions, the sample should remain unchanged for long periods. In particular, important impurities such as oxygen and water are not present. This technique has two disadvantages. First, even the most careful sealing-off procedure may lead to unwanted combustion impurities on the inside of the glass. Second, the thermocouple cannot easily be embedded in the sample; it must be attached to the outside of the tube. The second technique, used in this study, involves leaving the samples unsealed but with certain well-defined preparations. This has the advantage that the thermocouple can be embedded in the sample and temperature can be measured accurately. In the present case, the 8-mm diam samples were well closed-off with teflon tape. The NMR coil was $15 \mathrm{~mm}$ long and the thermocouple was $2 \mathrm{~mm}$ from the end of the coil well embedded in the finely powdered sample. By varying the position of the thermocouple it was determined that the gradient across the sample was never more than 1.5 kelvins. This technique has the disadvantage that impurities such as oxygen and water are available from the atmosphere. As in most laboratories we have traditionally used the former method but we find the problem of reliable and reproducible temperature measurement of utmost importance in these studies, especially when apparent or observed activation energies are large and $R$ is a rapidly changing function of $T$.

The DTBP was purchased from the Aldrich Chemical
Co. It was deuterated in the $\mathrm{OH}$ position by exchange with 2-propanol and purified by recrystallization from the same chemical. This resulted in a fluffy white powder and high-resolution NMR showed no hydrogen-bearing impurities in detectable quantities. Rather extensive experiments have shown that water vapor and oxygen are not playing an observable role $^{5}$ in DTBP. In particular, the relaxation is not being short circuited because of the presence of oxygen. This was determined, in our previous study, ${ }^{5}$ by comparing results from the samples of the degassed and sealed variety with those of the open variety. In that all the observed relaxation rates are greater than 1 $\mathrm{s}^{-1}$, it may not be surprising that oxygen is playing no role, but since the presence of the OD group might make the molecule more sensitive to impurities such as oxygen, it is important to study such possibilities. The thermal history of the sample, on the other hand, plays a more important role in determining the temperature dependence of $R$. The main consequence of uncontrolled thermal history is that the scatter in $R$ versus $T$ appears slightly larger as shown by the earlier $30-\mathrm{MHz}$ data in Fig. 2, but the general shape of $R$ versus $T$ appears unaffected. This scatter, which is well outside the experimental uncertainty in $R$, has been effectively eliminated in the present experiments at 8.5 and $53 \mathrm{MHz}$ by recycling the sample between 77 kelvins and room temperature 3 times before beginning each day's experiments. Once the three 77-300-kelvin cycles are done, reproducible results are obtained for greater than $12 \mathrm{~h}$ of experiments, regardless of the thermal history during the $12 \mathrm{~h}$.

The TTBZ was purchased from K\&K Laboratories and was very discolored by oxidation products. It was purified by recrystallization from ethanol to form a fluffy white powder. Since the temperature dependence of $R$ shows considerable unexpected structure, it was deemed important to establish its purity. High-resolution NMR confirmed that only tert-butyl groups and aromatic ring protons were present in detectable quantities and with the correct relative peak areas. However, given the uncertainties of our high-resolution NMR peak-area measurements, para-di-tert-butylbenzene (DTBZ), which is a starting material in one method of synthesis of TTBZ, ${ }^{8}$ could still have been present. We obtained ir spectra of both the TTBZ and DTBZ and, by comparing with the known spectra, ${ }^{9}$ confirmed that DTBZ was not present in detectable quantities. Although we have not studied sealed, degassed samples of TTBZ, the experience with MDBP and other related molecules ${ }^{3}$ convinces us that using the unsealed-tube technique along with the thermal recycling is best so long as the sample is fairly pure.

Since different frequencies may be investigated several months or even years apart, it is important to have longterm reproducibility for $R(\omega, \tau)$ measurements. Also, it is important to have accurate and reproducible temperature measurement, especially in molecular solids such as those studied here where $R$ is a rapidly changing function of temperature in some temperature regions. These two aspects lead to our choice of procedures: leaving the samples unsealed, inserting the thermocouple inside the sample, and thermally recycling the sample before each day's experiments. 


\section{DISCUSSION}

In organic molecular solids such as DTBP and TTBZ, the proton spin-lattice relaxation is caused by the modulation of the proton (hydrogen nuclei) dipole-dipole interactions by the reorientations of the $\mathrm{CH}_{3}$ and $\mathrm{C}\left(\mathrm{CH}_{3}\right)_{3}$ groups. Several molecules with pairs of $\mathrm{C}\left(\mathrm{CH}_{3}\right)_{3}$ groups in various positions in benzene rings (with and without $\mathrm{OH}$ groups) have been studied and a general classification of "types of rotors" has emerged. $6,7,10,11$ It can be concluded on the basis of these results and on the basis of the local symmetry of the isolated molecule that in both DTBP and TTBZ, the $\mathrm{C}\left(\mathrm{CH}_{3}\right)_{3}$ groups and their constituent $\mathrm{CH}_{3}$ groups are dynamically equivalent and reorient at the same mean rate. This assumes that the $\mathrm{OH}$ group in DTBP (a "second-nearest neighbor" on the ring) plays a negligible role in lowering the symmetry in that molecule. It is perhaps surprising that a $\mathrm{C}\left(\mathrm{CH}_{3}\right)_{3}$ group reorients at the same rate as its constituent $\mathrm{CH}_{3}$ group. This may occur because the $\mathrm{CH}_{3}$ groups must "gear" around the nearest-neighbor ring protons as the $\mathrm{C}\left(\mathrm{CH}_{3}\right)_{3}$ group reorients, although such classical pictures of reorientation must be treated with caution. This dynamical equivalence manifests itself in the observation of a single maximum in $R$, since this means that $\tau \sim \omega^{-1}$ occurs only once as temperature is varied. It follows from this discussion that the relaxation rate in DTBP and TTBZ is given by ${ }^{6}$

$$
R=\sum_{k=1}^{N} A^{(k)} \sum_{l=1}^{2} l^{2} J\left(l \omega, \tau^{(k)}\right),
$$

where the sum over $l$ accounts for the single and double spin flips allowed for in dipole-dipole interactions and the sum over $k$ accounts for all possible individual and superimposed reorientations. ${ }^{12}$ For both DTBP and TTBZ, $N=2 .^{6}$ The first term has $\tau^{(1)}=\tau$ which is the correlation time characterizing the reorientation of the methyls and the tert-butyls. The second term accounts for the superposition of the two motions and has $\tau^{(2)}=\tau / 2 .^{6}$ The strengths $A^{(k)}$ are squares of matrix elements of the nuclear-spin dipole-dipole interactions multiplied by geometrical factors. The $A^{(k)}$ can be calculated assuming given proton-proton interactions. If intramethyl and intermethyl, intra-(tert-butyl) interactions are considered, it can be shown ${ }^{6}$ that $A=A^{(1)}=A^{(2)}=2.29 \times 10^{9} \mathrm{~s}^{-2}$ for DTBP and $A=A^{(1)}=A^{(2)}=2.41 \times 10^{9} \mathrm{~s}^{-2}$ for TTBZ. These two numbers differ only in the different ratios of the numbers of methyl to total protons. (We emphasize that $A^{(1)}=A^{(2)}$ only to within about $1 \%$ and the equality is a coincidence. ${ }^{6}$ ) One would expect that these $A$ values are lower limits since all other dipole-dipole interactions are being neglected. Intramolecular inter-(tert-butyl) interactions should not matter because of the $r^{-6}$ dependence of $A$ where $r$ is the proton-proton distance. ${ }^{6}$ Intramolecular interactions between methyl protons and ring protons will contribute to a small extent, but the greatest unknown is the role of the intermolecular proton-proton, dipole-dipole interactions. Experimentally determined $A$ values range from near $0 \%$ to about $30 \%$ higher than values obtained from calculations based on the interactions discussed above. This reflects the varying degree of importance of intermolecular interactions in dif- ferent molecules. For the samples reported here, Eq. (1) becomes

$R=A[J(\omega, \tau)+4 J(2 \omega, \tau)+J(\omega, \tau / 2)+4 J(2 \omega, \tau / 2)]$.

The simplest spectral density $J(\omega, \tau)$ which could be used in Eq. (2) is

$$
J(\omega, \tau)=\frac{2 \tau}{1+\omega^{2} \tau^{2}} .
$$

This well-known, much-overused Lorentzian spectral density follows from the assumption of random, isotropic reorientation with a unique activation energy. ${ }^{1}$ It predicts that $\ln R$ versus $T^{-1}$ has slopes of opposite signs but equal magnitudes at high and low temperatures. It also has a single maximum in $\ln R$ for intermediate temperatures, but no other "structure" (dashed curve marked 2 in Fig. 2 or curves 2 and 3 in Fig. 3). It is therefore of limited use in interpreting data in systems such as those presented here. The $\ln R$ versus $T^{-1}$ in DTBP and TTBZ not only have quite different slopes at high and low temperatures, but have considerable structure. This is particularly true in TTBZ. It is important to note that the complex nature of $R$ versus $T$ in Figs. 2 and 3 does not arise because there are four terms in Eq. (2). Indeed, although we will use the full expression, Eq. (2), to fit the data, the fits are essentially indistinguishable from those using only the first term in Eq. (2); i.e., $R=A J(\omega, \tau)$. We use the full expression only because that allows us to compare the fitted parameter $A$ to the calculated one and thus learn about the role of intermolecular spin-spin interactions.

Relaxation data which cannot be fitted by a single Lorentzian spectral density are usually interpreted by assuming a superposition of Lorentzians, weighted by a distribution of correlation times, $\Lambda(\tau)$, which in turn can be obtained from a distribution $\Gamma(E)$ of activation energies. The two distributions are related by $\Lambda(\tau) d \tau=\Gamma(E) d E$ and by assuming the Arrhenius relation $\tau=\tau_{\infty} \exp (E / k T){ }^{4,13}$ With these assumptions, $J(\omega, \tau)$ may be written as

$$
\begin{aligned}
J(\omega, \tau) & =\int_{\tau_{\infty}}^{\infty} \Lambda\left(\tau^{\prime}\right) \frac{2 \tau^{\prime}}{1+\omega^{2}\left(\tau^{\prime}\right)^{2}} d \tau^{\prime} \\
& =\int_{0}^{\infty} \Gamma\left(E^{\prime}\right) \frac{2 \tau_{\infty} e^{E^{\prime} / k T}}{1+\omega^{2} \tau_{\infty}^{2} e^{2 E^{\prime} / k T}} d E^{\prime} .
\end{aligned}
$$

If the relaxation is characterized by a unique activation energy $E$ or a unique correlation time $\tau, \Gamma\left(E^{\prime}\right)=\delta\left(E^{\prime}-E\right)$ or $\Lambda\left(\tau^{\prime}\right)=\delta\left(\tau^{\prime}-\tau\right)$ and Eq. (4) yields the Lorentzian, Eq. (3).

Many of the spectral densities obtained using the above formalism ${ }^{13}$ can account for the slopes and the frequency dependence of $\ln R$ versus $1 / T$ at high and low temperatures. However, their structure at intermediate temperatures is usually similar to that of Eq. (3). A notable exception is a spectral density due to Frölich, ${ }^{14}$ obtained by assuming that the activation energy distribution is a constant, $\Gamma(E)=1 /\left(E_{h}-E_{l}\right)$ between a low-energy cutoff $E_{l}$ and a high-energy cutoff $E_{h}$, and zero otherwise. This results in relaxation-rate curves such as those shown by the dashed curves marked 1 in Figs. 2 and 3.

In the following, we extend Frölich's procedure and assume that $\Gamma(E)$ is given by a histogram consisting of $N$ 
boxes of which the $i$ th box has height $a_{i}$ and width $E_{i h}-E_{i l}$. We normalize $\Gamma(E)$ such that $\Sigma_{i} a_{i}\left(E_{i h}-E_{i l}\right)$ $=1$. It follows from Eq. (4) that the spectral density is given by

$J(\omega, \tau)=\frac{2 k T}{\omega} \sum_{i=1}^{N} \frac{1}{E_{i h}-E_{i l}}\left[\tan ^{-1}\left(\omega \tau_{i h}\right)-\tan ^{-1}\left(\omega \tau_{i l}\right)\right]$

with

$$
\tau_{i \rho}=\tau_{\infty} e^{E_{i \rho} / k T}
$$

for $\rho=l, h$ and $i=1$ to $N$.

Some microscopic models of the relaxation process result in a dependence of $\tau_{\infty}$ on $E$ or $T$ or both, ${ }^{15,16}$ but this dependence, if relevant, is usually sufficiently weak so that Eq. (6) is still an accurate representation of both the $E$ and $T$ dependence of the correlation time. ${ }^{16,17}$ In this study we take $\tau_{\infty}$ to be a constant.

Within the framework of a thermally activated random hopping model in which tunneling is not a significant factor, the $E$ represent barrier heights for a wide variety of rotational models. ${ }^{16}$ We note that if the distribution of barrier heights were known it could, in principle, be approximated to any accuracy by an appropriate histogram and the corresponding spectral density calculated in closed form using Eq. (5). We seek the simplest fits of the data. For both molecules, parameters were obtained by finding a "best fit" for the $\omega / 2 \pi=53-\mathrm{MHz}$ data. Fits for the other frequencies were then obtained by simply changing $\omega$ but otherwise using the same parameters. Thus the curves shown for 8.5 and $30 \mathrm{MHz}$ in Figs. 2 and 3 are fits with no adjustable parameters since they use parameters obtained from the 53- $\mathrm{MHz}$ fits. They should therefore be considered as predictions for these frequencies using the 53- $\mathrm{MHz}$ data.

The solid curves in Fig. 2 for DTBP were obtained using a histogram consisting of a flat background of height $a_{1}=0.51(\mathrm{kK})^{-1} \quad\left(\mathrm{kK} \equiv 10^{3}\right.$ kelvin $)$ with cutoffs of $E_{1 l}=0.87 \mathrm{kK}$ and $E_{1 h}=2.4 \mathrm{kK}$ and a sharp peak of height $a_{2}=227(\mathrm{kK})^{-1}$ centered at $E_{2}=1.78 \mathrm{kK}$, with $\left(E_{2 h}-E_{2 l}\right)=0.001 \mathrm{kK}$. The fitted value of $\tau_{\infty}$ was $7.4 \times 10^{-14} \mathrm{~s}$ and the fitted value of $A$ was $2.86 \times 10^{9} \mathrm{~s}^{-2}$. This value of $A$ is $25 \%$ greater than the calculated lowerlimit value. This is encouraging as it suggests that intermolecular dipole-dipole interactions, though present, are not dominating the relaxation. The width of the sharp peak can be made arbitrarily narrow so long as the area is kept constant at $a_{2}\left(E_{2 h}-E_{2 l}\right)=0.23$. It is essentially a Dirac $\delta$ function and the term in Eq. (5) corresponding to this could be replaced by Eq. (3). It is easily shown that this Lorentzian form follows from taking the limit $E_{2 h}-E_{2 l} \rightarrow 0$ in Eq. (5). The dashed lines labeled 1 and 2 in Fig. 2 show the contributions of the broad background and the $\delta$ function to $R$ at $53 \mathrm{MHz}$. This fit suggests that $77 \%$ of the magnetization is relaxing via the background distribution of activation energies, and $23 \%$ of the magnetization is relaxing with a single activation energy of $1.78 \mathrm{kK}$.

The simplest possible fits to the relaxation data for TTBZ all correspond to a broad background of activation energies and two sharp peaks. The fit shown in Fig. 3 corresponds to $a_{1}=0.26(\mathrm{kK})^{-1}$ with $E_{1 l}=0.93 \mathrm{kK}$ and $E_{1 h}=2.45 \mathrm{kK}$ (40\% of the magnetization), $a_{2}=40$ $(\mathrm{kK})^{-1}$ with $E_{2 l}=1.170 \mathrm{kK}$ and $E_{2 h}=1.175 \mathrm{kK}(20 \%$ of the magnetization), and $a_{3}=199(\mathrm{kK})^{-1}$ with $E_{3 l}=1.530$ $\mathrm{kK}$ and $E_{3 h}=1.532 \mathrm{kK}$ ( $40 \%$ of the magnetization). The dashed curve labeled 1 in Fig. 3 shows the contribution of the broad background while the curves marked 2 and 3 show the contributions of the spikes. The value of $\tau_{\infty}$ used was $8.0 \times 10^{-14} \mathrm{~s}$ and the parameter $A$ was $3.13 \times 10^{9} \mathrm{~s}^{-2}$, which is $30 \%$ larger than the calculated lower limit. Again, this fitted value for $A$ is encouraging and suggests that intermolecular dipole-dipole interactions, though significant, are not dominating the relaxation process.

These results are amenable to two levels of interpretation depending on whether one starts from the $\Gamma(E)$ 's that give the fits or the $\Lambda(\tau)$ 's that may be derived from them. The correlation-time distribution $\Lambda(\tau)$ may be used to define a nonexponential correlation function as a superposition of exponentials ${ }^{16}$ by

$$
\Phi(t)=\int_{\tau_{\infty}}^{\infty} \Lambda(\tau) e^{-t / \tau} d \tau
$$

The corresponding spectral density is obtained by taking the Fourier transform of $\Phi(t)$ resulting in the first form of Eq. (4). At the very least the analysis therefore leads to the determination of a correlation function that characterizes the relaxation process more accurately than those that are customarily used to fit relaxation data. ${ }^{6}$ On a less phenomenological level, the activation-energy distribution $\Gamma(E)$ may be used to give a statistical description of the local molecular environment. This interpretation, however, depends crucially on the assumption that the Arrhenius relation [Eq. (6)] with a constant $\tau_{\infty}$ accurately describes the $E$ and $T$ dependence of $\tau$. The results of Clough et al. ${ }^{17,18}$ provide support for this assumption.

The fits indicate activation-energy distributions characterized by one or two "spikes" superimposed on broad, flat backgrounds. The background distribution involves $77 \%$ of the DTBP molecules and $40 \%$ of the TTBZ molecules. It is interesting that the cutoff energies characterizing these two very different molecular solids are about the same: $E_{l} \sim 0.9 \mathrm{kK}$ and $E_{h} \sim 2.4 \mathrm{kK}$. One is tempted to suggest that this is a general phenomenon independent of the specific molecules and we are testing this in other molecular solids. In DTBP, the single spikes suggest that the rest of the tert-butyls are in the same environment yielding a single $E$ of $1.78 \mathrm{kK}$. In TTBZ, on the other hand, there are clearly two types of tert-butyl groups since there are spikes in the distribution at 1.17 and $1.53 \mathrm{kK}$. This suggests two "environments." Crystallographically inequivalent $\mathrm{CH}_{3}$ groups of this type occur in tetramethyl pyrazine $^{19}$ and tetramethyltin ${ }^{20}$ and crystallographically inequivalent $\mathrm{NH}_{4}$ groups occur in ammonium sulphate. $^{21}$ All three of these cases have been detected by nuclear-spin-relaxation techniques. Nuclear-spin-relaxation measurements cannot, by themselves, determine whether or not this is the case in TTBZ, and the measurements reported here must be complemented by $x$-ray studies or incoherent neutron scattering studies. ${ }^{20,21}$ 


\section{CONCLUSION}

The proton spin-lattice relaxation rates in DTBP and TTBZ cannot be characterized by a superposition of a finite number of Lorentzian spectral densities, each characterized by a unique activation energy. We find that the temperature and Larmor frequency dependence of the relaxation rate is well fitted by assuming a distribution $\Gamma(E)$ of activation energies consisting of one or two sharp spikes on a broad background ranging from about 0.9 to $2.3 \mathrm{kK}$. The interpretation in terms of activation-energy distributions hinges on the validity of the Arrhenius relation, $\tau=\tau_{\infty} \exp (E / k T)$, with a constant $\tau_{\infty}$. If the Arrhenius relation is not assumed, the analysis described here at least leads to the determination of a single nonexponential correlation function characterizing the relaxation.

Our studies are proceeding in several ways. We are continuing experiments in systems where only methyl group reorientations rather than superimposed methyl and tert-butyl group reorientations occur. We plan experi- ments at lower temperatures to aid in the investigation of low- $T$ cutoff energies in systems which do not show the equivalent of the low- $T$ slope such as that found in the molecules investigated here. On the theoretical side, we are investigating the generality of this "multibox" approach. This study suggests that the cutoffs and the spikes are important parameters in $\Gamma(E)$, but the sensitivity of $R$ versus $T$ to the detailed form of $\Gamma(E)$ between the cutoffs is less pronounced.

\section{ACKNOWLEDGMENTS}

We wish to thank Frank Mallory for helpful discussions. This work was supported, in part, by a Cottrell Research Grant to one of us (P.A.B.) from the Research Corporation and, in part, by a National Science Foundation Equipment Grant to P. A. B. (No. NSF-TFI-8102189). One of us (E.E.F.) acknowledges summer support from Shell Corporation. Part of this work fulfills partial requirements for the A. B. degree with Honors (Bryn Mawr College, 1983) for M. E. C. and F. A. F.
*Present address: Department of Physics, State University of New York at Stony Brook, Stony Brook, NY 11794.

${ }^{\dagger}$ Present address: Department of Materials Science and Engineering, Massachusetts Institute of Technology, Cambridge, MA 02139.

¥Present address: Department of Physics, Massachusetts Institute of Technology, Cambridge, MA 02139.

${ }^{1}$ A. Abragam, The Principles of Nuclear Magnetism (Oxford University Press, Oxford, 1961).

2P. L. Nordio and U. Segre, in The Molecular Physics of Liquid Crystals, edited by G. R. Luckhurst and G. W. Gray (Academic, New York, 1979).

${ }^{3}$ P. A. Beckmann, F. A. Fusco, and A. E. O'Neill, J. Magn. Reson. (in press).

${ }^{4}$ A. M. Albano, P. A. Beckmann, M. E. Carrington, F. A. Fusco, A. E. O'Neill, and M. E. Scott, J. Phys. C 16, L979 (1983).

${ }^{5}$ M. C. Aronson, P. A. Beckmann, B. J. Ross, and S. L. Tan, Chem. Phys. 63, 349 (1981).

6P. A. Beckmann, Chem. Phys. 63, 359 (1981).

${ }^{7}$ P. A. Beckmann, C. I. Ratcliffe, and B. A. Dunell, J. Magn. Reson. 32, 391 (1978).
${ }^{8}$ L. Ross, C. Barclay, and E. Betts, Can. J. Chem. 33, 672 (1955).

${ }^{9}$ C. J. Pouchert, The Aldrich Library of Infrared Spectra, Edition III (Aldrich Chemical Co., Milwaukee, 1981).

${ }^{10}$ C. I. Ratcliffe and B. A. Dunell, J. Chem. Soc. Faraday Trans. 2 73, 493 (1977).

11J. Yamauchi and C. A. McDowell, J. Chem. Phys. 75, 1051 (1981).

12P. A. Beckmann, Mol. Phys. 41, 1227 (1980).

${ }^{13}$ R. M. Hill and A. K. Jonscher, Contemp. Phys. 24, 75 (1983).

${ }^{14} \mathrm{H}$. Frölich, Theory of Dielectrics (Oxford University Press, Oxford, 1949).

${ }^{15}$ C. Brot, Chem. Phys. Lett. 3, 319 (1969).

${ }^{16}$ E. Helfand, J. Chem. Phys. 78, 1931 (1983).

${ }^{17}$ S. Clough and A. Heidermann, J. Phys. C. 13, 3585 (1980).

${ }^{18}$ S. Clough, A. Heidemann, A. J. Horsewill, J. D. Lewis, and M. N. J. Paley, J. Phys. C 15, 2495 (1982).

${ }^{19}$ S. Takeda and H. Chihara, J. Magn. Reson. 54, 285 (1983).

${ }^{20}$ M. Prager, K.-H. Duprée, and W. Müller-Warmuth, Z. Phys. B 51, 309 (1983).

${ }^{21}$ D. E. O'Reilly and T. Tsang, J. Chem. Phys. 46, 1291 (1967). 